\title{
IMPROVED CALCULATION OF PROLATE SPHEROIDAL RADIAL FUNCTIONS OF THE SECOND KIND AND THEIR FIRST DERIVATIVES
}

\author{
BY \\ ARNIE L. VAN BUREN AND JEFFREY E. BOISVERT \\ Naval Undersea Warfare Center, Newport, Rhode Island
}

\begin{abstract}
Alternative expressions for calculating the prolate spheroidal radial functions of the second kind $R_{m l}^{(2)}(c, \xi)$ and their first derivatives with respect to $\xi$ are shown to provide accurate values over wide parameter ranges where the traditional expressions fail to do so. The first alternative expression is obtained from the expansion of the product of $R_{m l}^{(2)}(c, \xi)$ and the prolate spheroidal angular function of the first kind $S_{m l}^{(1)}(c, \eta)$ in a series of products of the corresponding spherical functions. A similar expression for the radial functions of the first kind was shown previously to provide accurate values for the prolate spheroidal radial functions of the first kind and their first derivatives over all parameter ranges. The second alternative expression for $R_{m l}^{(2)}(c, \xi)$ involves an integral of the product of $S_{m l}^{(1)}(c, \eta)$ and a spherical Neumann function kernel. It provides accurate values when $\xi$ is near unity and $l-m$ is not too large, even when $c$ becomes large and traditional expressions fail. The improvement in accuracy using the alternative expressions is quantified and discussed.
\end{abstract}

1. Introduction. The scalar Helmholtz wave equation for steady waves, $\left(\nabla^{2}+k^{2}\right) \Psi=$ 0 , where $k=2 \pi / \lambda$ and $\lambda$ is the wavelength, is separable in the prolate spheroidal coordinates $(\xi, \eta, \varphi)$, with $1 \leq \xi \leq \infty,-1 \leq \eta \leq 1$, and $0 \leq \varphi \leq 2 \pi$. The factored solution is given by $\Psi_{m l}(\xi, \eta, \varphi)=R_{m l}(c, \xi) S_{m l}(c, \eta) \Phi_{m}(\varphi)$, where $R_{m l}(c, \xi)$ is the radial function, $S_{m l}(c, \eta)$ is the angular function, and $\Phi_{m}(\varphi)$ is the azimuthal function. Here $c=k a / 2$, where $a$ is the interfocal distance of the elliptic cross section of the spheroid. The radial function of the first kind $R_{m l}^{(1)}(c, \xi)$ and the radial function of the second kind $R_{m l}^{(2)}(c, \xi)$ are the two independent solutions to the second order radial differential equation resulting from the separation of variables. These solutions are dependent on four parameters $(m, l, c, \xi)$ and an eigenvalue (separation constant) $A_{m l}(c)$. Similarly, $S_{m l}^{(1)}(c, \eta)$ and $S_{m l}^{(2)}(c, \eta)$ are the two independent solutions to the second order angular

Received January 27, 2003.

2000 Mathematics Subject Classification. Primary 65D20; Secondary 33E10. 
differential equation resulting from the separation of variables. In the following discussion we assume that the degree $m$ is either zero or a positive integer, with $l$ equal to $m, m+1, m+2, \ldots$

Prolate spheroidal functions are used in solving boundary value problems of radiation, scattering, and propagation of scalar and vector acoustic and electromagnetic waves in prolate spheroidal coordinates. They also find application in the representation of bandlimited and time-limited physical processes.

Existing computer programs for calculating the prolate radial functions including those developed at the Naval Research Laboratory [1]-[3] utilize traditional expressions that fail to provide accurate results for some parameter ranges, especially at low values of $l-m$ when $c$ becomes large. In a previous paper [4], we showed that an alternative expression for calculating the prolate radial functions of the first kind provides accurate values for all parameter ranges. This expression follows directly from the product expression provided by Meixner and Schäfke [5, p. 307] that represents the product of the radial and angular functions in a series of products of the corresponding spherical Bessel and associated Legendre functions.

We show in this paper that the product expansion also leads to an alternative expression for $R_{m l}^{(2)}(c, \xi)$ that provides accurate values over most parameter ranges when $\xi$ is not near unity. The traditional spherical Neumann function expression for calculating $R_{m l}^{(2)}(c, \xi)$ is shown to be a special case of this alternative expression. We show also that the traditional associated Legendre function expression used to calculate $R_{m l}^{(2)}(c, \xi)$ when $\xi$ is near unity follows from the product expansion. We examine an expression for $R_{m l}^{(2)}(c, \xi)$ given by Flammer [6, p. 53] that involves an integral of the product of $S_{m l}^{(1)}(c, \eta)$ and a spherical Neumann function kernel. This expression is shown to provide accurate values when $\xi$ is near unity and $l-m$ is not too large, even when $c$ is large and the traditional associated Legendre expression fails. We describe some of the features of a new Fortran computer program we have developed to calculate the prolate spheroidal functions using both the traditional and the alternative expressions. We conclude the paper with a summary.

2. Angular functions of the first kind. The prolate angular function of the first kind $S_{m l}^{(1)}(c, \eta)$ is expressed [see for example ref. 6, p. 16] in terms of the corresponding associated Legendre functions of the first kind by

$$
S_{m l}^{(1)}(c, \eta)=\sum_{n=0,1}^{\infty} d_{n}(c \mid m l) P_{m+n}^{m}(\eta),
$$

where the prime sign on the summation indicates that $n=0,2,4, \ldots$ if $l-m$ is even or $n=1,3,5, \ldots$ if $l-m$ is odd. A three term recursion formula relates successive expansion coefficients $d_{n}, d_{n+2}$, and $d_{n+4}$ for given values of $l, m$, and $c$. Use of this formula to calculate the expansion coefficients requires a value for the separation constant or eigenvalue $A_{m l}(c)$, which is chosen to ensure nontrivial convergent solutions for $S_{m l}^{(1)}(c, \eta)$. The variational procedure developed by Bouwkamp [7] provides accurate values for both the eigenvalue and the ratios of successive coefficients $d_{n+2} / d_{n}$. Use of a 
starting value algorithm given in ref. [3] assures that the Bouwkamp procedure converges to the correct eigenvalue. We normalize the coefficients by requiring that $S_{m l}^{(1)}(c, \eta)$ has the same normalization factor as $P_{l}^{m}(\eta)$ [5], resulting in the following relation:

$$
\sum_{n=0,1}^{\infty} \frac{2(n+2 m) !}{[2(n+m)+1] n !}\left[d_{n}(c \mid m l)\right]^{2}=\frac{2(l+m) !}{(2 l+1)(l-m) !} .
$$

This normalization has the practical advantage of eliminating the need to numerically evaluate the normalization factor that is often encountered in problems involving expansions in spheroidal angular functions.

3. Expansion of the product of the radial and angular functions. The expansion of the product of $R_{m l}^{(2)}(c, \xi)$ and $S_{m l}^{(1)}(c, \eta)$ in terms of the corresponding spherical functions is given by:

$$
R_{m l}^{(2)}(c, \xi) S_{m l}^{(1)}(c, \eta)=\sum_{n=0,1}^{\infty} i^{n+m-l} d_{n}(c \mid m l) y_{n+m}(k r) P_{n+m}^{m}(\cos \theta) .
$$

This is a special case of the more general expansion given by Meixner and Schäfke [5, p. 307]. Using the relationship between the spherical coordinates $r$ and $\theta$ and spheroidal coordinates (about the same origin and with $\eta=1$ coincident with $\theta=0$ ), we obtain $k r=c\left(\xi^{2}+\eta^{2}-1\right)^{1 / 2}$ and $\cos \theta=\eta \xi /\left(\xi^{2}+\eta^{2}-1\right)^{1 / 2}$. Substituting for $S_{m l}^{(1)}(c, \eta)$ from (1) and solving for $R_{m l}^{(2)}(c, \xi)$ produces

$$
R_{m l}^{(2)}(c, \xi)=\frac{\sum_{n=0,1}^{\infty} i^{n+m-l} d_{n}(c \mid m l) y_{n+m}\left[c\left(\xi^{2}+\eta^{2}-1\right)^{1 / 2}\right] P_{n+m}^{m}\left[\eta \xi /\left(\xi^{2}+\eta^{2}-1\right)^{1 / 2}\right]}{\sum_{n=0,1}^{\infty} d_{n}(c \mid m l) P_{n+m}^{m}(\eta)} .
$$

The significance of this general expression is that it allows us to choose the value for $\eta$ that provides the maximum accuracy for calculated values of $R_{m l}^{(2)}(c, \xi)$.

4. Traditional spherical Neumann function expression. We consider the case when $\eta=1$. The argument of $P_{n+m}^{m}$ in both the numerator and the denominator approaches unity as $\eta$ approaches unity. Although $P_{n+m}^{m}$ approaches zero in this case for $m \neq 0$, the limit of the rhs of (4) exists and we obtain:

$$
R_{m l}^{(2)}(c, \xi)=\left(\frac{\xi^{2}-1}{\xi^{2}}\right)^{m / 2} \frac{\sum_{n=0,1}^{\infty} i^{n+m-l} d_{n}(c \mid m l) y_{n+m}(c \xi) \frac{(n+2 m) !}{n !}}{\sum_{n=0,1}^{\infty} d_{n}(c \mid m l) \frac{(n+2 m) !}{n !}} .
$$

Flammer [6, p. 32] derives (5) using integral representations of the spheroidal wave functions. The corresponding expression for the first derivative of $R_{m l}^{(2)}(c, \xi)$ with respect to $\xi$ is obtained by taking the first derivative of the rhs of (5). 
Equation (5) is the expression commonly used to calculate numerical values for $R_{m l}^{(2)}(c, \xi)$ when $\xi$ is not close to unity. Its numerator is an asymptotic series that is not absolutely convergent for any finite value of $c \xi$. However, this series often provides accurate values for $R_{m l}^{(2)}(c, \xi)$ if one takes the partial sum of the series up to and including the term where the relative contribution is a minimum (consecutive terms of the same sign are added and treated as a single term). It provides reasonably accurate results unless $c \xi$ is small or $\xi$ is close to unity or $c$ is large and $l-m$ is not large. Although the denominator series in (5) converges, it suffers subtraction errors when $c$ is large and $l-m$ is not large, i.e., the sum of the positive terms in the series becomes close in magnitude to the sum of the negative terms in the scries. The subtraction error is defined to be the number of accurate decimal digits that are lost in calculating the sum of the series. It is equal to the number of leading decimal digits that are the same in the positive and negative sums.

The subtraction error in the denominator series of (5) increases as $c$ increases. At a given value of $c$ and for $m=0$, the error is greatest at $l=0$, decreases as $l$ increases, and becomes negligible for $l$ somewhat larger than $2 c / \pi$. As $m$ increases for a given value of $c$, the error decreases at $l=m$ and falls off more slowly with increasing $l-m$. Figure 1 shows examples of this behavior. We note that for $c=40, m=0$, and $l=0$ the subtraction error is about 16 decimal digits. Increasing $c$ to 100 results in a subtraction error large enough to produce zero accuracy in the sum even when the calculations are performed in quadruple precision arithmetic (128 bits) with more than 30 decimal digits.

Figure 2 shows examples of the corresponding loss of accuracy in the numerator series of (5). The values plotted here are either the subtraction error in the partial sum or the number of decimal digits that are lost due to less than full convergence of the partial sum, depending on which is larger. We note that the loss in accuracy is comparable to that shown in Fig. 1 for the denominator.

Calculated values for $R_{m l}^{(2)}(c, \xi)$ and its first derivative with respect to $\xi$ have additional inaccuracy due to a lack of full accuracy in the $d_{n}$ cocfficients and the spherical Neumann functions used in their calculation. This inaccuracy can be limited to approximately two decimal digits with careful numerical technique.

5. Traditional associated Legendre function expression. We can use the product expansion for $R_{m l}^{(2)}(c, \xi)$ and the angular function of the second kind $S_{m l}^{(2)}(c, \eta)$ in terms of the corresponding spherical functions to obtain:

$$
R_{m l}^{(2)}(c, \xi)=\frac{\sum_{n=-\infty}^{\infty} i^{n+m-l} d_{n}(c \mid m l) y_{n+m}\left[c\left(\xi^{2}+\eta^{2}-1\right)^{1 / 2}\right] Q_{n+m}^{m}\left[\eta \xi /\left(\xi^{2}+\eta^{2}-1\right)^{1 / 2}\right]}{S_{m l}^{(2)}(c, \eta)} .
$$

Here the summation must be taken from $-\infty$ to $+\infty$ since the associated Legendre functions of the second kind $Q_{n+m}^{m}$ do not vanish for $n$ less than zero as $P_{n+m}^{m}$ does. 


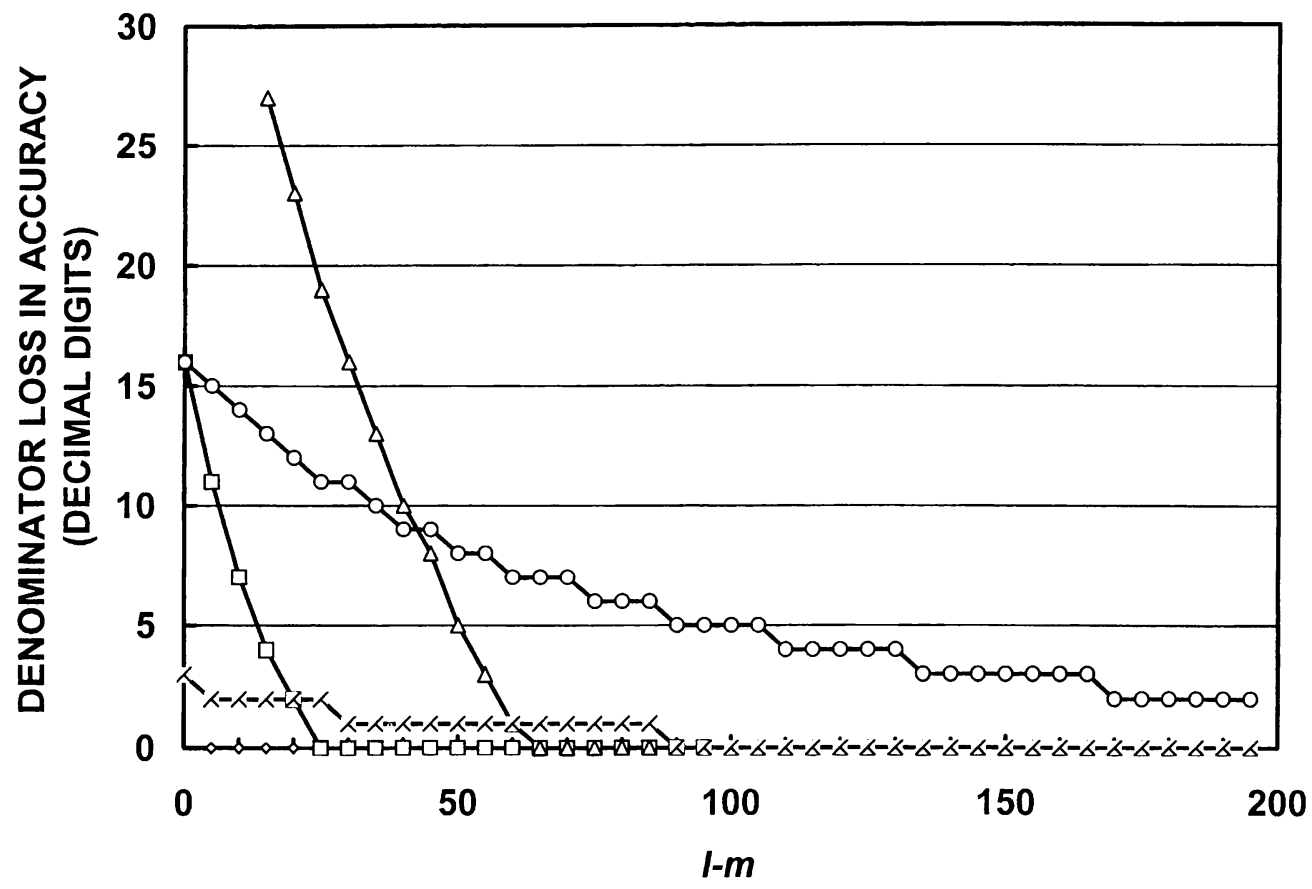

FIG. 1. Subtraction error in decimal digits in the denominator of the traditional expressions (5) and (10) plotted versus $l-m$ for selected parameters $(c, m): \diamond(10,0) ; \square(40,0) ; \times(40,100) ; \triangle(100,0)$; $\circ(100,100)$.

Taking the limit of the rhs of (6) as $\eta$ approaches infinity produces:

$$
R_{m l}^{(2)}(c, \xi)=\frac{\sum_{n=-\infty}^{\infty} \lim _{\eta \rightarrow \infty}\left[i^{n+m-l} y_{n+m}(c \eta)\right] d_{n}(c \mid m l) Q_{n+m}^{m}(\xi)}{\lim _{\eta \rightarrow \infty} S_{m l}^{(2)}(c, \eta)} .
$$

The phase term $i^{n+m-l}$ is real and equal to $(-1)^{(n+m-l) / 2}$ since $n$ has the same parity as $l-m$. Using the asymptotic properties of $y_{n+m}$ and the requirement that the radial functions are defined to have the same asymptotic limit as the corresponding spherical functions, we obtain:

$$
\lim _{\eta \rightarrow \infty}\left[i^{n+m-l} y_{n+m}(c \eta)\right]=\lim _{\eta \rightarrow \infty} y_{l}(c \eta)=\lim _{\eta \rightarrow \infty} R_{m l}^{(2)}(c, \eta) .
$$

Equation (7) now becomes:

$$
R_{m l}^{(2)}(c, \xi)=\lim _{\eta \rightarrow \infty}\left[R_{m l}^{(2)}(c, \eta) / S_{m l}^{(2)}(c, \eta)\right] \sum_{n=-\infty}^{\infty} d_{n}(c \mid m l) Q_{n+m}^{m}(\xi) .
$$

Since $R_{m l}^{(2)}(c, \xi)$ and $S_{m l}^{(2)}(c, \eta)$ satisfy the same differential equation, they are proportional to each other. The term in brackets is thus independent of $\eta$. It is defined to be the reciprocal of the joining factor of the second kind $\kappa_{m l}^{(2)}(c)$. Useful expressions for $\kappa_{m l}^{(2)}(c)$ 


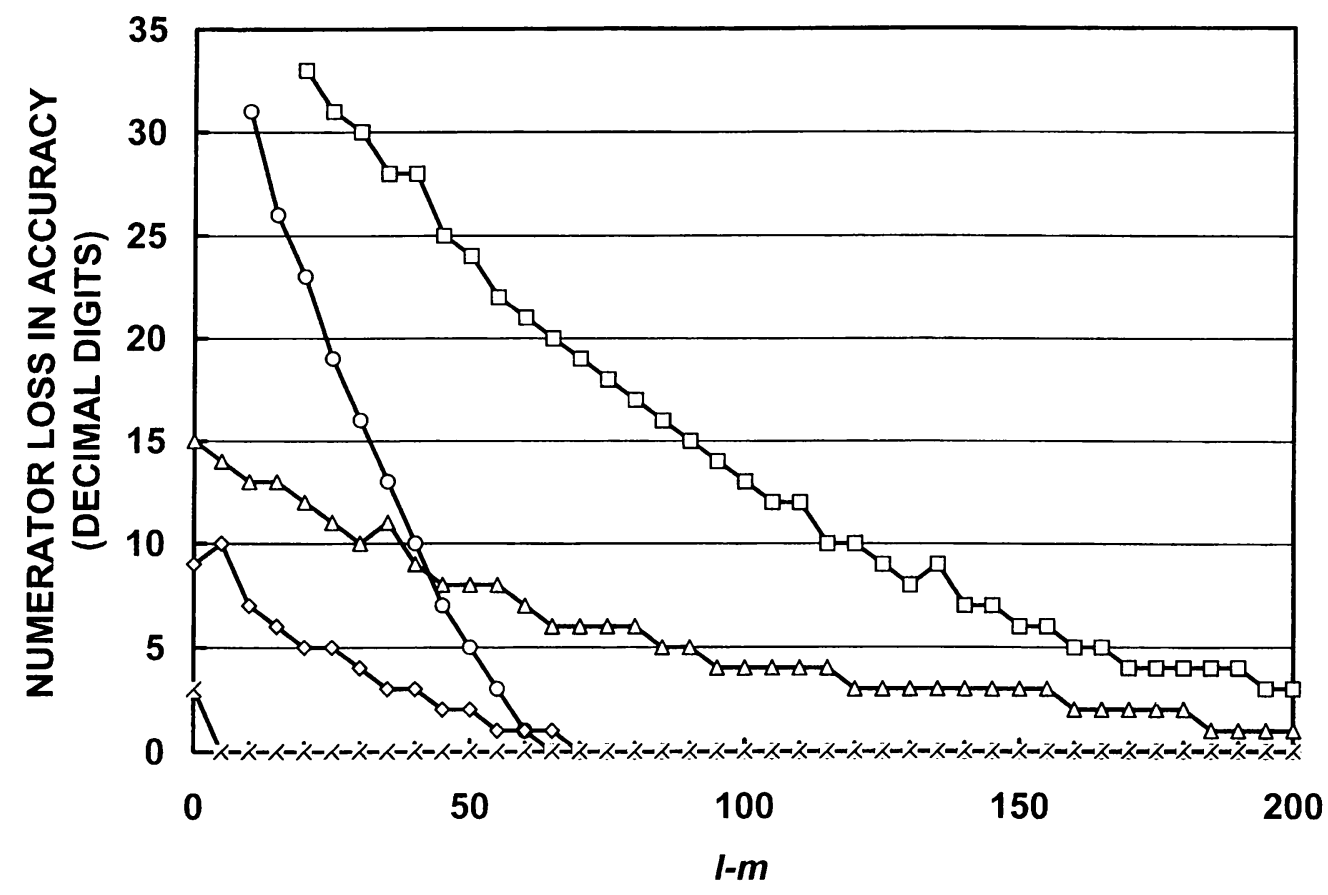

Fic. 2. Loss of accuracy in decimal digits due to both subtraction error and lack of full convergence in the numerator of the spherical Neumann function expression (5) plotted versus $l-m$ for selected parameters $(\xi, c, m): \times(1.1,10,0) ; \circ(2,100,0) ; \diamond(2,100,100)$; $\square(2,200,100) ; \triangle(10,100,100)$.

are found in Flammer [6, p. 33]. We thus have the following expression:

$$
R_{m l}^{(2)}(c, \xi)=\frac{1}{\kappa_{m l}^{(2)}(c)} \sum_{n=-\infty}^{\infty} d_{n}(c \mid m l) Q_{n+m}^{m}(\xi) .
$$

Of course, we could have used the fact that $R_{m l}^{(2)}(c, \xi)$ and $S_{m l}^{(2)}(c, \eta)$ are proportional to each other to obtain (10) directly without using the product expansion.

Although $Q_{n+m}^{m}(\xi)$ becomes infinitely large when $n$ is less than $-2 m$, its product with $d_{n}$ is finite and proportional to $P_{-n-m-1}^{m}(\xi)$. The rhs of (10) then divides into two series, one over $n$ from $-2 m$ (or $-2 m+1$ if $l-m$ is odd) to $\infty$ involving $Q_{n+m}^{m}(\xi)$ and one over $n$ from $2 m+2$ (or $2 m+1$ if $l-m$ is odd) to $\infty$ involving $P_{n-m-1}^{m}(\xi)$. The result is the traditional associated Legendre function expression used to evaluate $R_{m l}^{(2)}(c, \xi)$ when $\xi$ is near unity [see, e.g., ref. 6]. The joining factor $\kappa_{m l}^{(2)}(c)$ contains the same series that is given in the denominator of the traditional Neumann function expression (5). Thus the denominator of the associated Legendre function expression (10) suffers loss in accuracy for low values of $l-m$ similar to that shown in Fig. 1 due to subtraction errors that increase without bound as $c$ increases. In Fig. 3 we show examples of the corresponding loss in accuracy due to subtraction errors in the numerator of (10). The results are comparable to those for the denominator. 


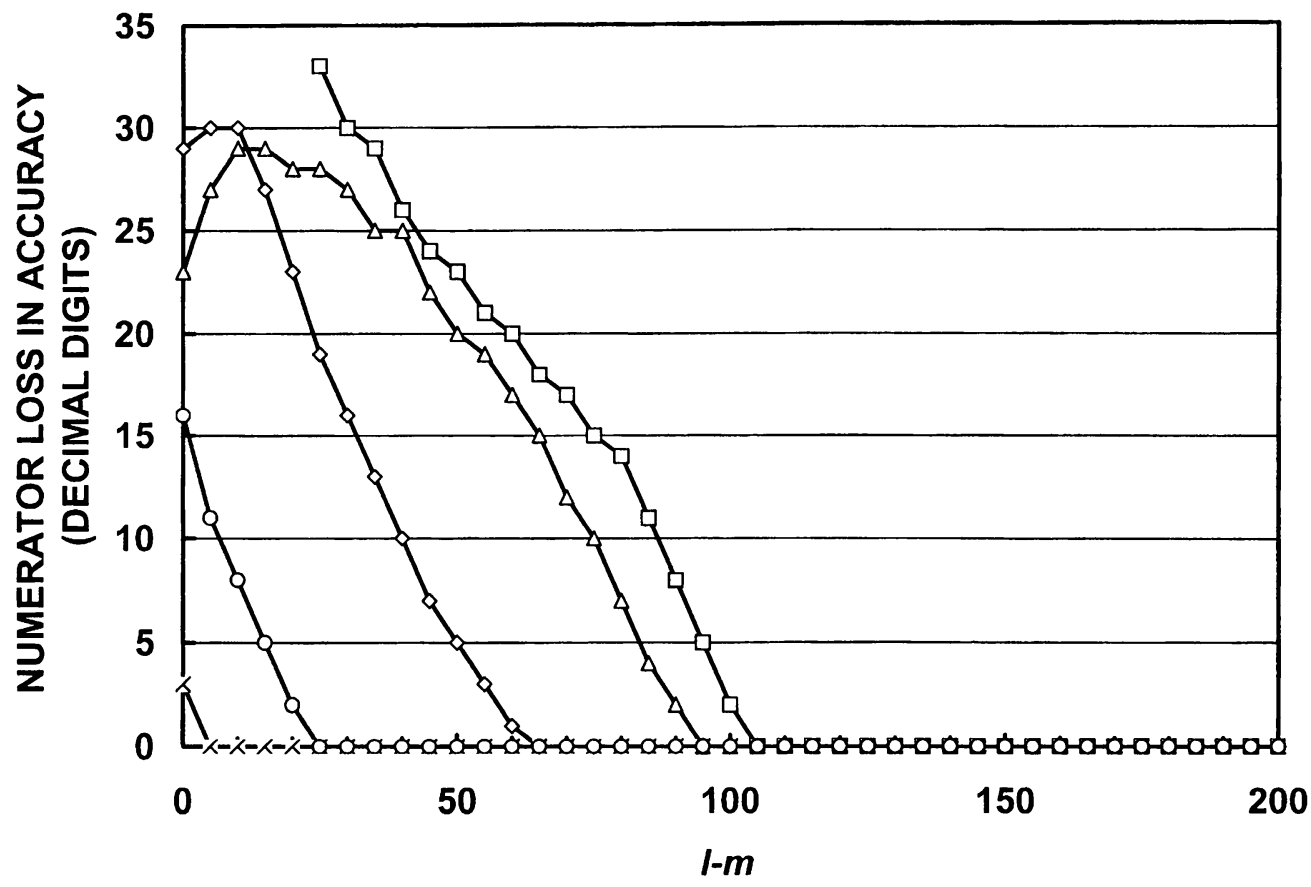

FIG. 3. Loss of accuracy in decimal digits due to subtraction error in the numerator of the associated Legendre function expression (10) plotted versus $l-m$ for selected parameters $(\xi, c, m) ; \diamond(1.00000001,100,0) ; \triangle(1.00000001,100,100)$; $\times(1.001,10,0) ; \circ(1.001,40,0) ; \square(1.1,100,0)$.

6. An alternative variable $\eta$ expression for calculating $R_{m l}^{(2)}(c, \xi)$. We now consider the expression (4) for $R_{m l}^{(2)}(c, \xi)$ in the case where $0 \leq \eta \leq 1$. In Ref. 4 we examined the corresponding expression for $R_{m l}^{(1)}(c, \xi)$ given by the rhs of (4) with the spherical Neumann function $y_{n+m}\left[c\left(\xi^{2}+\eta^{2}-1\right)^{1 / 2}\right]$ replaced with the spherical Bessel function $j_{n+m}\left[c\left(\xi^{2}+\eta^{2}-1\right)^{1 / 2}\right]$. We showed there that the expression obtained by taking the limit of the rhs as $\eta$ approaches zero is numerically robust, providing accurate values for $R_{m l}^{(1)}(c, \xi)$ over all parameter ranges with no subtraction error unless $\xi$ is near a root. The equivalent expression for $R_{m l}^{(2)}(c, \xi)$ obtained from the limit of (4) as $\eta$ approaches zero is unfortunately not as robust. However, it does provide accurate values at low values of $l-m$ unless $c \xi$ is small or $\xi$ is close to unity, even when $c$ is large and the traditional spherical Neumann expression (5) fails. Nearly full accuracy is obtained over a range of values of $l$ extending from $m$ to some nominal value. The accuracy then decreases monotonically toward zero with increasing $l$.

To estimate the accuracy of calculated values for $R_{m l}^{(2)}(c, \xi)$ and the first derivative, we compare the theoretical value for the Wronskian

$$
R_{m l}^{(1)} \frac{d R_{m l}^{(2)}}{d \xi}-R_{m l}^{(2)} \frac{d R_{m l}^{(1)}}{d \xi}=\frac{1}{c\left(\xi^{2}-1\right)}
$$




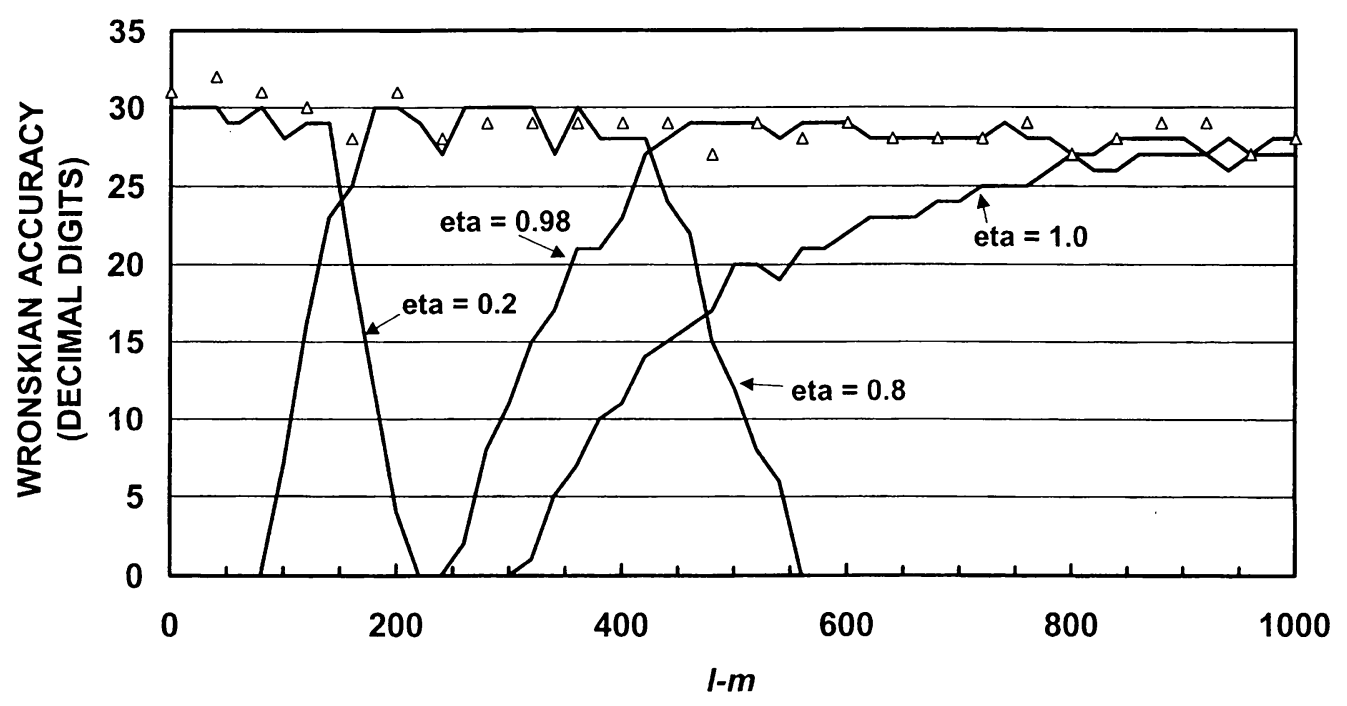

FIG. 4. Wronskian accuracy in decimal digits obtained for $\xi=1.35$, $c=500$, and $m=100$ when using the variable $\eta$ expression (4) plotted versus $l-m$ for selected values of $\eta$.

with its value computed from numerical values for the radial functions and their first derivatives. Accurate values for $R_{m l}^{(1)}(c, \xi)$ and its first derivative are available using the expressions and methods described in ref. 4 . The number of decimal digits of agreement between the theoretical and computed Wronskian is then a measure of the accuracy of $R_{m l}^{(2)}(c, \xi)$ and its first derivative.

When we evaluate (4) with $\eta$ chosen to be somewhat larger than zero, say equal to 0.4 , we find that the resulting accuracy for $R_{m l}^{(2)}(c . \xi)$ is lower for $l=m$ than was obtained when $\eta=0$. However, the accuracy increases with increasing $l$ until a maximum in accuracy is obtained. This maximum is nearly full accuracy when $\xi$ is not near unity. It is somewhat less than full accuracy when $\xi$ is near unity especially when both $c$ and $m$ are very large. The maximum in accuracy is maintained for a range of $l$ values, and then the accuracy decreases toward zero at values of $l$ somewhat higher than for $\eta=0$. Evaluating (4) with progressively larger values of $\eta$ (up to unity) results in progressively higher values of $l$ where a local maximum in accuracy is obtained.

An example of this behavior is shown in Fig. 4 for $\xi=1.35, c=500$, and $m=100$. Calculations were performed on a personal computer in quadruple precision arithmetic with 33 decimal digits. Only a few values of $\eta$ are necessary to provide at least 25 digits of Wronskian accuracy over the full range of $l$. We have also plotted in Fig. 4 the Wronskian accuracy obtained using the new computer program profen (see Sec. 8). Profen increments $\eta$ as $l$ increases in order to maintain a specified minimum number of decimal digits of accuracy, assuming that this accuracy is achievable. The results shown here were computed with 27 decimal digits specified.

In Fig. 5 we show corresponding results for $\xi=1.1, c=500$, and $m=100$. Here there are no values of $\eta$ that provide a Wronskian accuracy greater than 20 digits when 


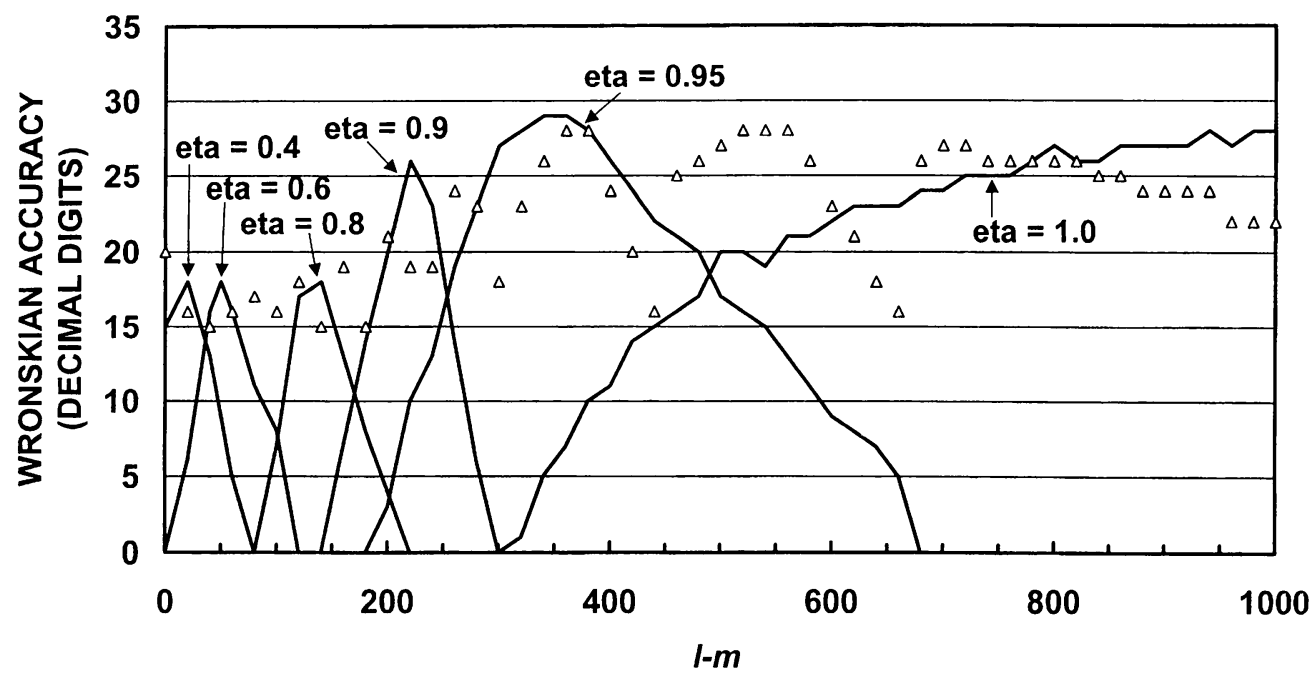

FIG. 5. Wronskian accuracy in decimal digits obtained for $\xi=1.1$, $c=500$, and $m=100$ when using the variable $\eta$ expression (4) plotted versus $l-m$ for selected values of $\eta$.

$l-m$ is less than about 150 . The accuracy curves for individual values of $\eta$ are much narrower than those for $\xi=1.35$ except for $\eta$ near unity. We also show in Fig. 5 the Wronskian accuracy obtained using profen with 15 digits specified. Profen continues to use the same value of $\eta$ as $l$ increases until the accuracy falls to 15 when it then searches for a higher value of $\eta$ where the accuracy is 16 or greater. As $l-m$ increases, the resulting accuracy curve exhibits a number of peaks and valleys as it sequentially follows the rise and fall in Wronskian accuracy for progressively larger values of $\eta$. Of course, 25 or more digits of accuracy can be obtained for values of $l-m$ greater than about 200 through use of different values of $\eta$ than were selected in profen. However, this would increase the computation time and probably offers no practical value since most of the lower order function values do not have more than 18 digits of accuracy.

7. Integral expression for calculating $R_{m l}^{(2)}(c, \xi)$. When $\xi$ is close to unity, $c$ is large, $m$ is not small, and $l-m$ is not large, both the traditional expressions as well as the variable $\eta$ expression described above provide values for $R_{m l}^{(2)}(c, \xi)$ with significantly reduced accuracy. The resulting function values, even when computed using quadruple precision arithmetic, can have insufficient accuracy. We have discovered that the following integral expression given by Flammer [6] is useful for this case:

$$
\begin{aligned}
& R_{m l}^{(2)}(c, \xi)=\frac{(-1)^{(l-m) / 2}(2 m+1)}{2^{m+1} m ! d_{0}(c \mid m l)} \\
& \quad \times \int_{-1}^{+1}\left[\frac{\left(\xi^{2}-1\right)\left(1-\eta^{2}\right)}{\left(\xi^{2}+\eta^{2}-1\right)}\right]^{m / 2} y_{m}\left[c\left(\xi^{2}+\eta^{2}-1\right)^{1 / 2}\right] S_{m l}^{(1)}(c, \eta) d \eta, \quad l-m \text { even. }
\end{aligned}
$$




$$
\begin{aligned}
& R_{m l}^{(2)}(c, \xi)=\frac{(-1)^{(l-m-1) / 2}(2 m+3)}{2^{m+1} m ! d_{1}(c \mid m l)} \\
& \quad \times \int_{-1}^{+1} \frac{\left[\left(\xi^{2}-1\right)\left(1-\eta^{2}\right)\right]^{m / 2}}{\left(\xi^{2}+\eta^{2}-1\right)^{(m+1) / 2}} \xi \eta y_{m+1}\left[c\left(\xi^{2}+\eta^{2}-1\right)^{1 / 2}\right] S_{m l}^{(1)}(c, \eta) d \eta, \quad l-m \text { odd } .
\end{aligned}
$$

It is convenient to define $z=c\left(\xi^{2}+\eta^{2}-1\right)^{1 / 2}$ and a window function $F_{m}(\xi, \eta)$ given by

$$
F_{m}(\xi, \eta)=\left[\frac{\left(\xi^{2}-1\right)\left(1-\eta^{2}\right)}{\xi^{2}+\eta^{2}-1}\right]^{m / 2} .
$$

In (12) and (13) we use (1) to expand $S_{m l}^{(1)}(c, \eta)$ in terms of associated Legendre functions and obtain:

$$
\begin{aligned}
& R_{m l}^{(2)}(c, \xi)=B_{m l}^{(a)}(c) \sum_{n=0}^{\infty} d_{n}(c \mid m l) I_{m n}^{(a)}(c, \xi), \quad l-m \text { even }, \\
& R_{m l}^{(2)}(c, \xi)=\xi B_{m l}^{(b)}(c) \sum_{n=1}^{\infty} d_{n}(c \mid m l) I_{m n}^{(b)}(c, \xi), \quad l-m \text { odd }
\end{aligned}
$$

where $B_{m l}^{(a)}(c)$ is the leading coefficient in (12), $B_{m l}^{(b)}(c)$ is the leading coefficient in (13), and

$$
\begin{gathered}
I_{m n}^{(a)}(c, \xi)=\int_{-1}^{+1} F_{m}(\xi, \eta) y_{m}(z) P_{m+n}^{m}(\eta) d \eta, \quad l-m \text { even, } \\
I_{m n}^{(b)}(c, \xi)=c \int_{-1}^{+1}\left[F_{m}(\xi, \eta) / z\right] \eta y_{m+1}(z) P_{m+n}^{m}(\eta) d \eta, \quad l-m \text { odd } .
\end{gathered}
$$

The series on the rhs of (15) and (16) are both well behaved. They converge rapidly with negligible subtraction error when $l-m$ is not large and $\xi$ is near unity, even when $c$ becomes extremely large.

We obtain corresponding expressions for the first derivatives of $R_{m l}^{(2)}(c, \xi)$ with respect to $\xi$ from (12) and (13) by differentiating, utilizing standard recursion relations for the spherical Neumann functions, and collecting terms. This gives:

$$
\begin{aligned}
& \frac{d R_{m l}^{(2)}}{d \xi}(c, \xi)=\frac{m \xi}{\xi^{2}-1} R_{m l}^{(2)}(c, \xi)-\frac{(-1)^{(l-m) / 2}(2 m+1) c \xi}{2^{m+1} m ! d_{0}(c \mid m l)} \\
& \quad \times \int_{-1}^{+1} \frac{\left[\left(\xi^{2}-1\right)\left(1-\eta^{2}\right)\right]^{m / 2}}{\left(\xi^{2}+\eta^{2}-1\right)^{(m+1) / 2}} y_{m+1}\left[c\left(\xi^{2}+\eta^{2}-1\right)^{1 / 2}\right] S_{m l}^{(1)}(c, \eta) d \eta, \quad l-m \text { even } \\
& \frac{d R_{m l}^{(2)}}{d \xi}(c, \xi)=\frac{(m+1) \xi^{2}-1}{\xi\left(\xi^{2}-1\right)} R_{m l}^{(2)}(c, \xi)-\frac{(-1)^{(l-m-1) / 2}(2 m+3) c \xi^{2}}{2^{m+1} m ! d_{1}(c \mid m l)} \\
& \quad \times \int_{-1}^{+1} \frac{\left[\left(\xi^{2}-1\right)\left(1-\eta^{2}\right)\right]^{m / 2}}{\left(\xi^{2}+\eta^{2}-1\right)^{(m+2) / 2}} \eta y_{m+2}\left[c\left(\xi^{2}+\eta^{2}-1\right)^{1 / 2}\right] S_{m l}^{(1)}(c, \eta) d \eta, \quad l-m \text { odd }
\end{aligned}
$$


Replacing $S_{m l}^{(1)}(c, \eta)$ with its expansion in (1) we obtain:

$$
\begin{gathered}
\frac{d R_{m l}^{(2)}}{d \xi}(c, \xi)=\frac{m \xi}{\xi^{2}-1} R_{m l}^{(2)}(c, \xi)-c \xi B_{m l}^{(a)} \sum_{n=0}^{\infty} d_{n}(c \mid m l) I_{m n}^{(c)}(c, \xi), \quad l-m \text { even, } \\
\frac{d R_{m l}^{(2)}}{d \xi}(c, \xi)=\frac{(m+1) \xi^{2}-1}{\xi\left(\xi^{2}-1\right)} R_{m l}^{(2)}(c, \xi)-c \xi^{2} B_{m l}^{(b)} \sum_{n=1}^{\infty} d_{n}(c \mid m l) I_{m n}^{(d)}(c, \xi), \quad l-m \text { odd },
\end{gathered}
$$

where

$$
\begin{aligned}
I_{m n}^{(c)}(c, \xi) & =\int_{-1}^{+1}\left[F_{m}(\xi, \eta) / z\right] y_{m+1}(z) P_{m+n}^{m}(\eta) d \eta, \quad l-m \text { even } \\
I_{m n}^{(d)}(c, \xi) & =\int_{-1}^{+1}\left[F_{m}(\xi, \eta) / z^{2}\right] \eta y_{m+2}(z) P_{m+n}^{m}(\eta) d \eta, \quad l-m \text { odd }
\end{aligned}
$$

The series on the rhs of (21) and (22) converge rapidly with negligible subtraction error.

The required integrals $I_{m n}(c, \xi)$ have an integrand that is symmetric about $\eta=0$. They can be computed using Gauss quadrature over positive values of $\eta$ and doubling the result. However, one must be careful to increase the density of quadrature points near $\eta=0$ when $\xi$ approaches unity because of the singularity of the spherical Neumann functions at $z=0$. The integrals tend to decrease in magnitude as $n$ increases, the decrease accompanied by loss of accuracy from increasing subtraction error. This causes a decrease in accuracy in both $R_{m l}^{(2)}(c, \xi)$ and its first derivative as $l-m$ increases and limits the integration method to non-large values of $l-m$.

We have derived relations between the different integrals through use of recursion relations for the associated Legendre functions. For example, we can replace $\eta P_{m+n}^{m}(\eta)$ in the rhs of $(24)$ with its equivalent in terms of $P_{m+n-1}^{m}(\eta)$ and $P_{m+n+1}^{m}(\eta)$ and obtain the following relation:

$$
(2 n+2 m+1) I_{m n}^{(b)}=(n+2 m) I_{m, n-1}^{(c)}+(n+1) I_{m, n+1}^{(c)} .
$$

Thus the integrals $I_{m n}^{(b)}$ can be calculated directly from (25) instead of computing them using Gauss quadrature. Other relations we have derived are not as useful for calculating radial functions with a given degree $m$ since they relate integrals of one kind and degree $m$ to integrals of a second kind and degree $m \pm 1$. These could, however, be useful when one is computing the radial functions for a range of $m$ values.

The window function $F_{m}(\xi, \eta)$ given in (14) is equal to unity at $\eta=0$ and decreases monotonically to zero as $\eta$ increases to 1 . Its rate of decrease with respect to $\eta$ increases as $m$ increases, especially when $\xi$ is near unity. A large rate of decrease effectively lowers the upper limit of the range of integration. With a smaller range of integration, fewer oscillations of the integrand contribute. The result is that the integrals decrease in magnitude more slowly (and lose accuracy) more slowly with increasing $n$. This allows the integral expression to be used to higher values of $l-m$ when $m$ is large and $\xi$ is near unity.

In Figures 6 and 7 we show examples of the accuracy obtained using the integral expression. Here the Wronskian accuracy is plotted versus $l-m$ for selected combinations 


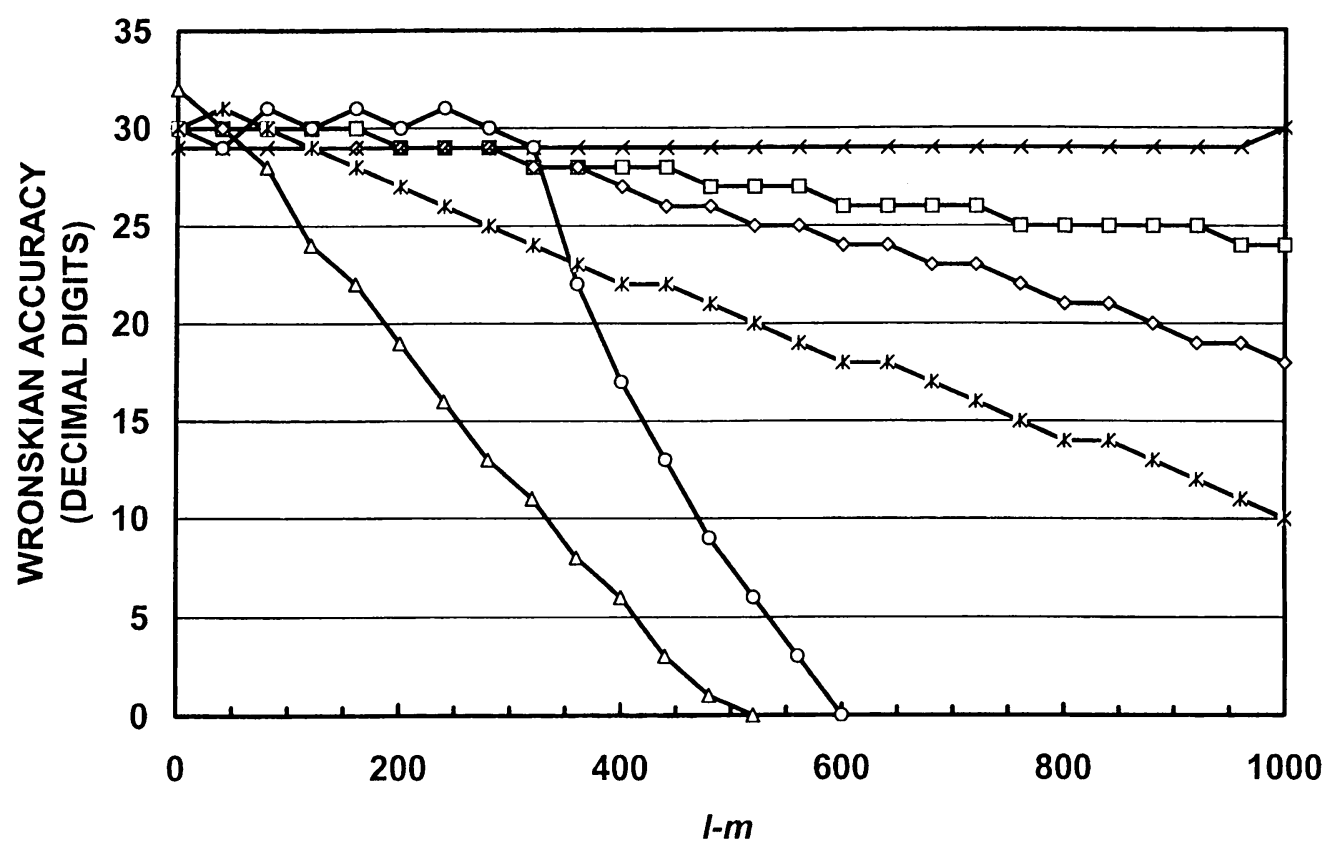

FIG. 6. Wronskian accuracy in decimal digits obtained using the integral expressions (12), (13), (19), and (20) plotted versus $l-m$ for selected parameters $(\xi, c, m): \square(1.0001,100,0) ; \times(1.0001,500,300)$; $*(1.001,100,0) ; \triangle(1.01,100,0) ; \circ(1.01,500,0) ; \diamond(1.01,500,300)$.

of $\xi, c$, and $m$. Results are given for $\xi=1.01,1.001$, and 1.0001 in Fig. 6 and for $\xi=1.05$ and 1.1 in Fig. 7. We see that in general the integral expression tends to provide accurate results to higher values of $l-m$ when $\xi$ is closer to unity. When $\xi$ is less than about 1.05 and $c$ is large, the accuracy for fixed $l-m$ tends to increase with increasing $m$. When $\xi$ is greater than about 1.05, this is true only if $m$ is not small or $l-m$ is large.

8. A computer program for calculating the prolate spheroidal functions. We have developed a Fortran computer program called profen to calculate the prolate spheroidal functions. Profcn performs calculations in quadruple precision arithmetic with $30+$ decimal digits available and runs on a personal computer equipped with a Fortran compiler that supports quadruple precision arithmetic. Calculation options include (1) radial functions of the first kind and their first derivatives, (2) radial functions of both the first and second kind and their first derivatives, (3) angular functions of the first kind, and (4) angular functions of the first kind and their first derivatives. If desired, both radial and angular functions can be calculated during the same run. Profcn provides an estimate of the number of accurate digits in the function values based either on the Wronskian (when both $R_{m l}^{(1)}$ and $R_{m l}^{(2)}$ are calculated and the Wronskian is not used to determine the denominator for variable $\eta$ calculations) or on the calculation of the subtraction and convergence errors introduced into the results. The output of profen includes diagnostic 


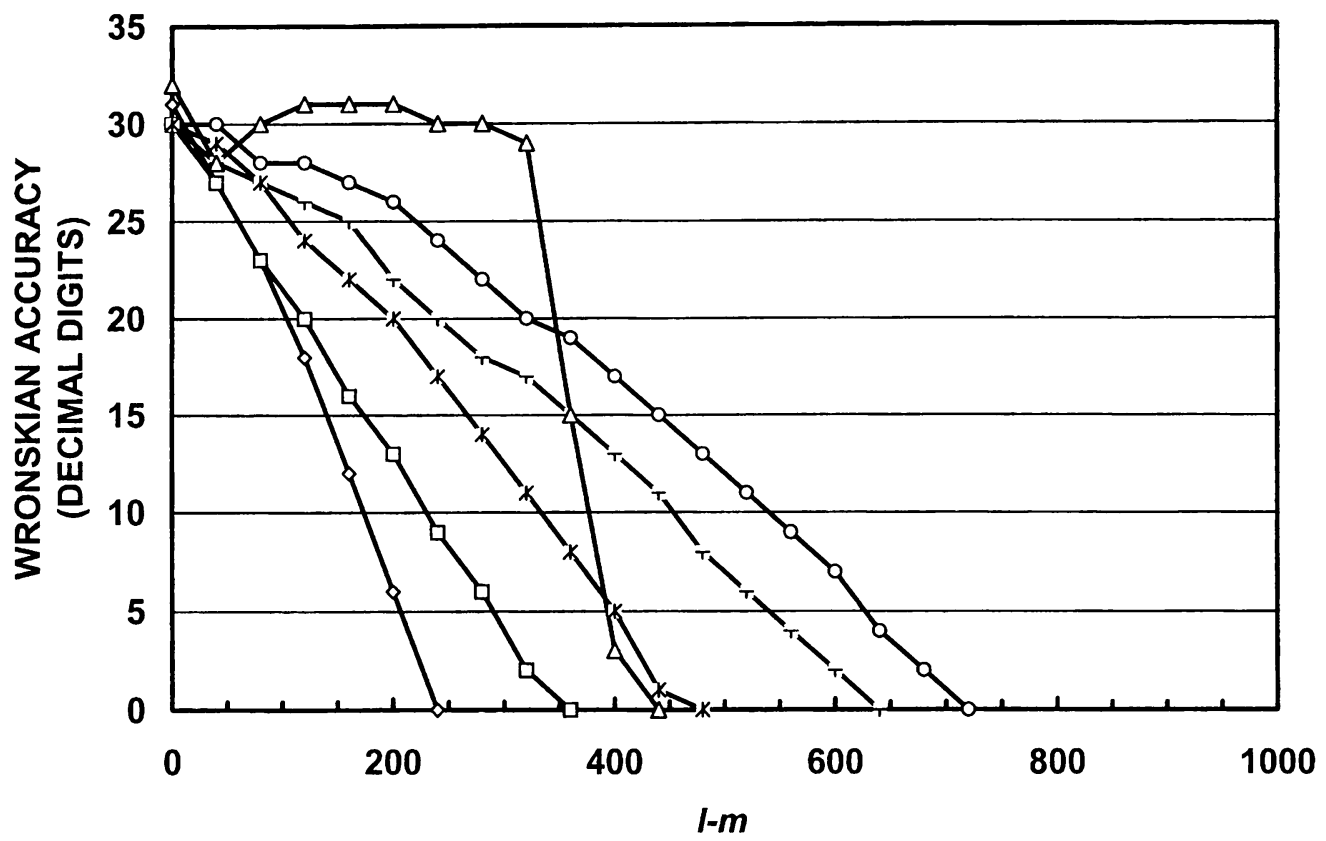

FIG. 7. Wronskian accuracy in decimal digits obtained using the integral expressions (12), (13), (19), and (20) plotted versus $l-m$ for selected parameters $(\xi, c, m): \circ(1.05,100,300) ; \triangle(1.05,500,0)$; $+(1.05,500,300) ; *(1.1,100,300) ; \diamond(1.1,500,100) ; \square(1.1,500,300)$.

files (one for radial functions and one for angular functions) including information such as the number of terms both available and used in the various series.

Profcn calculates the angular functions of the first kind using (1) and the radial functions of the first kind using the new method described in ref. [4]. It calculates the radial functions of the second kind using either the traditional spherical Neumann function expression (5), the traditional associated Legendre function expression (10), the variable $\eta$ expression (4), or the integral expressions in (12) and (13). One specifies the number of accurate decimal digits that are desired. The expression chosen for a specific calculation depends on the input parameters and the desired accuracy. For small to moderate values of $c$, profcn uses the traditional Neumann function expression for values of $\xi$ greater than 1.4 and the traditional associated Legendre function expression elsewhere. For larger values of $c$, it uses the integral expressions, starting with $l=m$ and continuing until the maximum desired value for $l$ is reached or the Wronskian accuracy has decreased to the number of accurate decimal digits desired. In the latter case profen also calculates $R_{m l}^{(2)}(c, \xi)$ using a second method beginning with the next value of $l$. It uses a traditional expression when the subtraction error in the denominator of the Neumann function expression is sufficiently small. If this provides the desired accuracy, the integral expressions are abandoned in favor of the traditional expression. If the traditional expression is unable to provide the desired accuracy, the variable $\eta$ expression is tried. 
Here profen tries values of $\eta$ beginning near zero and increasing toward unity using a variable step size algorithm. Usually a value for $\eta$ is found after a few steps that provides the desired accuracy. This value of $\eta$ is used for succeeding values of $l$ until the accuracy falls below the desired accuracy. Then profen increments $\eta$ until the desired accuracy is obtained. The process is repeated as necessary. Whenever the desired accuracy cannot be obtained, the algorithm converges to the value of $\eta$ that gives the highest possible accuracy for that value of $l$. Profen switches to a traditional expression when it provides sufficiently accurate results. It determines this by testing the appropriate traditional expression every ten values of $l$ once the subtraction error in the denominator of the Neumann expression is sufficiently small.

Our calculations using profen have shown that the use of the integral expression together with the traditional expressions provides at least 10 digits of accuracy (when using quadruple precision arithmetic) for $\xi \leq 1.001$ when $c \leq 1000$ and for $1.001 \leq \xi \leq 1.01$ when $c \leq 500$. Ten digits should be sufficient accuracy for most applications. When we include the variable $\eta$ expression, we obtain at least ten digits of accuracy for all values of $\xi$ when $c \leq 1000$ except for values of $\xi$ between 1.005 and 1.15 where fewer than 10 digits (as few as zero) may be obtained when $c$ is greater than about 400 and $m$ is between about 30 and 120. We tested the expressions for the first 1001 values of $l-m$, unless $c$ was larger than 500 where we tested for the first $2 c+1$ values. Few if any applications will require spheroidal function values for $l-m$ larger than this, although the traditional expressions should provide accurate values if required.

We have improved this situation significantly by using the fact that the numerator in the variable $\eta$ expression (4) can be significantly more accurate than the denominator for a narrow range of $\eta$ values. This is also true for the corresponding expression for the first derivative, which has the same denominator as (4). We can then use the Wronskian expression (11) to solve for the value of the denominator in (4) using values for the corresponding numerators of both $R_{m l}^{(2)}(c, \xi)$ and its first derivative and calculated accurate values for $R_{m l}^{(1)}(c, \xi)$ and its first derivative. This results in the following expression:

$$
R_{m l}^{(2)}=\frac{\mathbb{N}\left(R_{m l}^{(2)}\right)}{c\left(\xi^{2}-1\right)\left[R_{m l}^{(1)} \mathbb{N}\left(d R_{m l}^{(2)} / d \xi\right)-\left(d R_{m l}^{(1)} / d \xi\right) \mathbb{N}\left(R_{m l}^{(2)}\right)\right]},
$$

where $\mathbb{N}(y)$ denotes the numerator value of $y$.

Use of (26) and the corresponding expression for the first derivative of $R_{m l}^{(2)}(c, \xi)$ often provides improved accuracy when insufficient accuracy is obtained otherwise. Of course, we can no longer use the Wronskian expression (11) to estimate the resulting accuracy. We instead estimate the accuracy using the degree of convergence and associated subtraction errors of the two numerators. We now obtain at least ten digits of accuracy for all values of $\xi$ when $c \leq 1000$ except for a few values of $l-m$ near 500 when $m$ lies between about 50 and 120 and $\xi$ is between about 1.008 and 1.015 . Here as few as 5 digits may be obtained. We have successfully computed accurate values for values of $c$ significantly larger than 1000 and obtain ten or more digits except for a narrow range of values for $l$ when $\xi$ is near 1.01 and $m$ is moderate to large. In all cases we computed the spheroidal functions for values of $l-m$ up to 1000 or $2 c$, whichever is larger. 
9. Summary. We have identified alternative expressions for calculating the prolate spheroidal radial functions of the second kind $R_{m l}^{(2)}(c, \xi)$ and their first derivatives with respect to $\xi$ that provide accurate values over wide parameter ranges where the traditional expressions fail to do so. The variable $\eta$ expression is based on the expansion of the product of $R_{m l}^{(2)}(c, \xi)$ and the prolate spheroidal angular function of the first kind $S_{m l}^{(1)}(c, \eta)$ in a series of products of the corresponding spherical functions. This expression provides accurate values over most parameter ranges when $c$ is not small and $\xi$ is not near unity. We showed that both the traditional Neumann function expression and the traditional associated Legendre function expression can be obtained from the product expansion. The second alternative expression for calculating $R_{m l}^{(2)}(c, \xi)$ involves an integral of the product of $S_{m l}^{(1)}(c, \eta)$ and a spherical Neumann function kernel. It can be computed efficiently to provide accurate values when $\xi$ is near unity and $l-m$ is not too large, even when $c$ becomes large and traditional expressions fail. We also briefly described a new Fortran computer program profcn that utilizes both traditional expressions and alternative expressions to compute the prolate spheroidal radial functions of both kinds and their first derivatives and the prolate spheroidal angular functions of the first kind and their first derivatives.

Acknowledgments. This work was supported by the Office of Naval Research Code 321 and the Naval Undersea Warfare Center ILIR Program.

\section{REFERENCES}

[1] B. J. King, R. V. Baier, and S. Hanish, A Fortran computer program for calculating the prolate spheroidal radial functions of the first and second kind and their first derivatives, Naval Research Lab. Rpt. 7012 (1970)

[2] B. J. King and A. L. Van Buren, A Fortran computer program for calculating the prolate and oblate angle functions of the first kind and their first and second derivatives, Naval Research Lab. Rpt. 7161 (1970)

[3] B. J. Patz and A. L. Van Buren, A Fortran computer program for calculating the prolate spheroidal angular functions of the first kind, Naval Research Lab. Memo. Rpt. 4414 (1981)

[4] A. L. Van Buren and J. E. Boisvert, Accurate calculation of prolate spheroidal radial functions of the first kind and their first derivatives, Quart. Appl. Math. 60, 589-599 (2002)

[5] J. Meixner and F. W. Schäfke, Mathieusche Funckionen und Sphäroidfunckionen, Springer-Verlag, Berlin, 1954

[6] C. Flammer, Spheroidal Wave Functions, Stanford Univ. Press, Stanford, Calif., 1957

[7] C. J. Bouwkamp, Theoretical and numerical treatment of diffraction through a circular aperture, IEEE Trans. Antennas and Propagation AP-18, 152 176 (1970)

[8] M. Abramowitz and C. A. Stegun, Handbook of Mathematical Functions with Formulas, Graphs, and Mathematical Tables, 9th printing, Dover, New York, NY, 1972 